\title{
CII-DC-AdTRAIL cell gene therapy inhibits infiltration of CII-reactive $T$ cells and CII-induced arthritis
}

\author{
Zhongyu Liu, ${ }^{1}$ Xin Xu, ${ }^{1}$ Hui-Chen Hsu, ${ }^{1}$ Albert Tousson, ${ }^{2}$ Ping-Ar Yang, ${ }^{1}$ Qi Wu, ${ }^{1}$ \\ Cunren Liu, ${ }^{1}$ Shaohua Yu, ${ }^{1}$ Huang-Ge Zhang, ${ }^{1,3}$ and John D. Mountz ${ }^{1,3}$
}

${ }^{1}$ Department of Medicine, Division of Clinical Immunology and Rheumatology, and

${ }^{2}$ Department of Cell Biology, The University of Alabama at Birmingham, Birmingham, Alabama, USA

${ }^{3}$ Veterans Administration Medical Center (VAMC), 700 South 19th Street, Birmingham, Alabama, USA

\begin{abstract}
Previously, we described an APC-adenovirus (APC-Ad) FasL cell gene therapy method which could be used to deplete autoreactive T cells in vivo. FasL was toxic, however, and controlled regulation of FasL was not achieved. Here we describe an improved approach to delivering TNF-related apoptosis-inducing ligand (TRAIL) in vivo in which collagen II-induced (CII-induced) arthritis-susceptible (CIA-susceptible) DBA/ $1 \mathrm{j}$ mice were treated with CII-pulsed DCs that had been transfected with a novel Ad system. The Ad was engineered to exhibit inducible TRAIL under the control of the doxycycline-inducible (DOX-inducible) tetracycline response element (TRE). Four groups of mice were treated with CII-DCAdTRAIL+DOX, CII-DC-AdTRAIL (no DOX), CII-DC-AdGFP+DOX, or DC-AdTRAIL+DOX (no CII), beginning 2 weeks after priming with CII in CFA. The incidence of arthritis and infiltration of $\mathrm{T}$ cells in the joint was significantly decreased in CII-DC-AdTRAIL+DOX-treated mice. The in vitro splenic $\mathrm{T}$ cell proliferative response and induction of IFN- $\gamma$ to bovine CII stimulation were also significantly reduced in mice treated with CII-DC-AdTRAIL+DOX. AdTRAIL+DOX was not toxic to DCs or mice but could induce activated $T$ cells to undergo apoptosis in the spleen. Our results suggest that CII-DCAdTRAIL+DOX cell gene therapy is a safe and effective method for inhibiting the development of CIA.

J. Clin. Invest. 112:1332-1341 (2003). doi:10.1172/JCI200319209.
\end{abstract}

\section{Introduction}

Arthritis in DBA/ $1 \mathrm{j}$ mice induced with bovine type II collagen (CII) is a prototype model of rheumatoid arthritis (RA) and shares many clinical and histopathological similarities to RA (1-4). Synovitis and erosions of cartilage and bone are hallmarks of CII-induced arthritis (CIA), and susceptibility to both RA and CIA is linked to the expression of specific MHC class II molecules $(2,5-8)$.The disease progression of CIA has been proposed to be associated with production of murine CII autoantibodies (9-11). Cumulative evidence further

Received for publication June 16, 2003, and accepted in revised form September 16, 2003.

Address correspondence to: John D. Mountz, The University of Alabama at Birmingham, Department of Medicine, Division of Clinical Immunology and Rheumatology, 701 South 19th Street, Lyons-Harrison Research Building 473, Birmingham, Alabama 35294, USA. Phone: (205) 934-8909; Fax: (205) 975-6648;

E-mail: john.mountz@ccc.uab.edu.

Huang-Ge Zhang and John D. Mountz contributed equally to this work.

Conflict of interest: The authors have declared that no conflict of interest exists.

Nonstandard abbreviations used: rheumatoid arthritis (RA); type II collagen (CII); CII-induced arthritis (CIA); antigen (Ag); generalized lymphoproliferative disease ( $g l d)$; adenovirus (Ad); tetracycline response element (TRE); TNF-related apoptosisinducing ligand (TRAIL); death receptor (DR); doxycycline (DOX); reverse tetracycline transactivator (rtTA); room temperature (RT); 3,3-diaminobenzidine (DAB); DCs transfected with AdTRAIL (DC-AdTRAIL). suggests that $\mathrm{CD}^{+} \mathrm{T}$ cell-mediated autoimmune responses play a critical role in the pathogenesis of RA (12-17). CIA can be transferred using $\mathrm{CD}^{+} \mathrm{T}$ cells, and the cytokine profile in CIA is consistent with the Th1 profile (18-23). Furthermore, T cells from CIA mice can transfer disease into SCID mice that are subsequently boosted with CII peptide. These results suggest that the generation of $\mathrm{T}$ cells specific for the CII epitopes presented by APCs is an important pathogenic mechanism of CIA (24-26).

A major challenge in the development of effective therapies for the treatment of RA is finding a method for the specific inhibition of the inflammatory disease processes without induction of generalized immunosuppression $(4,27,28)$. Because APCs play a central role in defining antigen $(\mathrm{Ag})$ specificity, they provide an access point for specific manipulation of the immune system. We have previously described techniques for modifying APCs so that they express specific Ag's along with FasL and have demonstrated that these modified APCs deleted only those $\mathrm{T}$ cells that recognize the specific Ag without causing general immunosuppression $(29,30)$. We previously demonstrated that treatment of mycoplasmas-infected B6-generalized lymphoproliferative disease $(g l d / g l d)$ mice with adenovirus (Ad) FasLtransfected (AdFasL-transfected) APCs derived from Fas-deficient $l p r$ mice ( $l p r$-APC-AdFasL) resulted in a significantly decreased incidence of chronic arthritis (31). We also have shown previously that normal APCs can be 
used in combination with an Ad system expressing an inducible FasL, providing there is coexpression of the p35 apoptosis inhibitor. For inducible expression, FasL was placed under the control of the tetracycline response element (TRE). The treatment with CII-APCAd FasLp35Tet effectively prevents CII-primed DBA/ $1 \mathrm{j}$ mice from developing arthritis without impairing the host immune response to an irrelevant Ag OVA (16).

TNF-related apoptosis-inducing ligand (TRAIL) is a type II membrane protein of the TNF superfamily. TRAIL can potentially interact with two cell-surface death receptors (DR), DR4 and DR5. Although TRAIL is involved in multiple processes, the precise roles of TRAIL in health and disease are unknown. TRAIL is more effective than FasL in the induction of apoptosis in some types of tumor cells but less toxic to normal cells than FasL (32-34). Both TRAIL and TRAIL receptors are constitutively expressed in various tissues and are upregulated upon cell activation $(35,36)$. TRAIL-deficient mice are hypersensitive to CIA and streptozotocin-induced diabetes and develop heightened autoimmune responses (37). Chronic blockade of TRAIL in mice with soluble DR5 exacerbated autoimmune arthritis, and intraarticular TRAIL gene transfer ameliorated the disease (38, 39). In vivo, TRAIL blockade led to profound hyperproliferation of synovial cells and arthritogenic lymphocytes and heightened the production of cytokines and autoantibodies. In vitro, TRAIL inhibited DNA synthesis and prevented cell cycle progression of lymphocytes. Thus, unlike other members of the TNF superfamily, TRAIL is a prototype inhibitor protein that inhibits autoimmune inflammation by inducing apoptosis and by blocking cell cycle progression (38).

To achieve Ag-specific $\mathrm{T}$ cell deletion in a regulatable manner, we developed a binary adenovirus system, which permits doxycycline-inducible (DOXinducible) expression of TRAIL under the control of the DOX-inducible TRE and a second Ad that expressed rtTA. CII-injected DBA/ $1 \mathrm{j}$ mice, which developed CII arthritis, were treated with the CIIpulsed DCs that had been transfected with this binary Ad system. AdTRAIL+DOX was not toxic to DCs. Treatment with CII-DC-AdTRAIL+DOX significantly suppressed the $T$ cell infiltration and development of CIA in the joint. Furthermore, $\mathrm{T}$ cell proliferation and IFN- $\gamma$ induction were dramatically reduced in the group of mice treated with CII-DC-AdTRAIL+DOX.

\section{Methods}

Mice. Female homozygous DBA $/ \mathrm{j}$ mice ( 7 weeks old) were obtained from The Jackson Laboratory (Bar Harbor, Maine, USA). All mice were kept in a room equipped with an air-filtering system. The cages, bedding, water, and food were sterilized, and the mice were handled with sterile gloves. All animal procedures were approved by The University of Alabama at Birmingham Institutional Animal Care and Use Committee.

Ad expression vectors for inducible TRAIL and GFP. An adenovirus expressing inducible TRAIL was construct- ed as described previously $(29,40,41)$. Briefly, the fulllength TRAIL was first cloned into the BamHI polylinker site of a TRE vector (Clontech, Palo Alto, California, USA) site. The TRE-regulated TRAIL fragment, including the bovine growth hormone poly-A tail, was then excised with XhoI and HindIII, followed by insertion into the Klenow-filled NotI site of the pShuttleCMV, leading to the production of pShuttleTRAIL. The recombinant Ad AdTRAIL was produced by in vitro recombination of pShuttle TRAIL with pAdeasy 1 as described previously (42). AdTRAIL was produced in 293 cells as described elsewhere (40). Using the reverse tetracycline transactivator (rtTA), recombinant AdCMVrtTA was constructed as described previously (30) (construct generously provided by J.B. Uney, University of Bristol, Bristol, United Kingdom) (43) to allow expression of the rtTA, thereby enabling DOX-inducible expression of TRAIL. A DOX inducible AdGFP binary Ad system was developed as described above.

Induction of arthritis. DBA/ $1 \mathrm{j}$ female mice were $\mathrm{immu}$ nized at 7 weeks of age at the base of the tail with 200 $\mu \mathrm{g}$ of bovine CII dissolved in $100 \mu \mathrm{l}$ of $0.05 \mathrm{M}$ acetic acid and mixed with an equal volume $(100 \mu \mathrm{l})$ of CFA (Chondrex Inc., Redmond, Washington, USA).

Isolation of $D C$. Bone marrow was collected from both the femurs and tibias of DBA $/ 1 \mathrm{j}$ mice at 8 weeks of age. Bone marrow cells were incubated with a mixture of Ab's directed against B220 (clone RA3-3A1/6.1), CD4 (clone GK1.5), CD8 (clone 53-6.72), and Ia (B21-2), using supernatants from hybridomas (American Type Culture Collection, Manassas, Virginia, USA) for 30 minutes on ice. $\mathrm{Ab}$-coated cells were removed using goat anti-rat IgG magnetic beads (Biosource International, Camarillo, California, USA) to eliminate T cells, B cells, NK cells, and granulocytes. The remaining cells were cultured in RPMI-1640 supplemented with 10\% FBS, 1\% L-glutamine, $1 \%$ penicillin, $50 \mu \mathrm{M}$ 2-mercaptoethanol, $10 \mathrm{mM}$ HEPES ( $\mathrm{pH} 7.4$ ), and $5 \mathrm{ng} / \mathrm{ml}$ of recombinant mouse GM-CSF (PeproTech Inc., Rocky Hill, New Jersey, USA). After 4 days of culture, loosely adherent DC clusters were collected and replated in 100-mm dishes. The DCs were then cultured for 12 hours with $10 \mathrm{ng} / \mathrm{ml}$ of LPS (SigmaAldrich, St. Louis, Missouri, USA) to induce maturation. The purity of the analysis of DCs was established by the expression of CD80, CD86, and CD11c, indicating that more than $85 \%$ of cells were mature DCs.

Transfection of DCs with Ad. Immature DCs were generated as described above. The DCs and HT1080 cells were transfected with AdTRAIL or AdGFP at $50 \mathrm{pfu} / \mathrm{cell}$ of each virus system for 1 hour. After washing, the DCs were cultured for 12 hours with LPS, then washed again. For in vitro analysis, the cells were treated with different concentrations of DOX (Sigma-Aldrich) for an additional 24 hours. For in vivo experiments, the DCs were intraperitoneally transferred $\left(5 \times 10^{6} \mathrm{cells} / \mathrm{mouse}\right)$ into mice.

In vitro analysis of $D C s$ after transfection with $A d$. The infection rate of Ad into DCs was determined using a fluorescence microscope (BX41; Olympus Optical Co., Tokyo, Japan). Expression of functional TRAIL on the 
DCs was evaluated by incubation with DCs or TRAILsensitive HT1080 fibrosarcoma cells with different concentrations of DOX for an additional 24 hours. The in vitro cytotoxicity of the DCs or HT1080 cells was estimated using an ATPlite assay.

In vivo analysis of DCs after transfection with $A d . \mathrm{DBA} / 1 \mathrm{j}$ mice immunized with bovine $\mathrm{CII}$ were intraperitoneally injected with $5 \times 10^{6}$ DCs transfected with AdTRAIL or AdGFP as described above. The expression of TRAIL was induced by the addition of DOX $(2 \mathrm{mg} / \mathrm{ml})$ with $4 \%$ sucrose into the drinking water. Forty-eight hours later, the spleen and liver were collected and embedded with paraffin and OCT. For mice receiving AdGFPtransfected DCs, the spleen, liver, and axillary and inguinal lymph nodes were frozen and sectioned, then counterstained with Hoechst, and GFP-positive cells were identified using a fluorescence microscope (BX41; Olympus Optical Co.).

Induction of TRAIL-mediated apoptosis in spleen. Apoptosis in the spleen and liver were evaluated using in situ TUNEL staining (Oncogene Research Products, Cambridge, Massachusetts, USA). The same tissues as collected above were sectioned. The slides were then incubated with fresh proteinase $\mathrm{K}(20 \mu \mathrm{g} / \mathrm{ml})$ at room temperature (RT) for 15 minutes. The endogenous peroxidases were inactivated by incubating the slides with $3 \% \mathrm{H}_{2} \mathrm{O}_{2}$ at RT for 5 minutes. After washing with $\mathrm{H}_{2} \mathrm{O}$, Klenow enzyme was added to the slides. The slides were then incubated at $37^{\circ} \mathrm{C}$ for 1 hour in a humidified chamber. Nonspecific staining was blocked by incubating the slides with 5\% BSA at RT for 30 minutes. The slides were then incubated with a peroxidase-conjugated streptavidin at a 1:50 dilution in 5\% BSA/PBS buffer at RT for 30 minutes after washing six times with PBS, the slides were incubated with 3,3-diaminobenzidine (DAB) at RT for 7 minutes for color development. Apoptotic cells were identified by the dark brown staining of the nuclei. Counterstaining was done with methyl green at RT for 3 minutes.

Treatment protocols for mice immunized with CII. To determine the role of DC-AdTRAIL (DCs transfected with AdTRAIL) cell gene therapy in CIA, the effects of the following four treatment protocols were compared: (a) CIIDC-AdTRAIL (no DOX), (b) CII-DC-AdGFP+DOX, (c) DC-AdTRAIL+DOX (no CII pulse), and (d) CII-DCAdTRAIL+DOX. To delete the CII-reactive $\mathrm{T}$ cells, mature DCs from the bone marrow of DBA/ $1 \mathrm{j}$ mice were pulsed with $\mathrm{T}$ cell proliferation-grade ArthrogenCIA CII (Chondrex Inc.) as described by the manufacturer, then transfected with a novel Ad system. The DCs were injected intraperitoneally into mice at a dose of $5 \times 10^{6}$ cells per mouse. The Ad was engineered to exhibit DOX-inducible expression of TRAIL under the control of the DOX-inducible TRE. DCs transfected with this AdTRAIL express murine TRAIL in a DOXinducible manner. Four groups of mice were treated with either CII-DC-AdTRAIL+DOX or, for control groups, with CII-DC-AdTRAIL (no DOX), CII-DCAdGFP+DOX, or DC-AdTRAIL+DOX (no CII pulse), beginning 2 weeks after in vivo priming with CII in CFA twice per week for 2 weeks. Induction of TRAIL on these DCs was accomplished in three of these groups by the addition of DOX $(2 \mathrm{mg} / \mathrm{ml})$ or $0.3 \%$ eyhanol as a control to the drinking water with $4 \%$ sucrose for 10 weeks starting at the time of administration of DC-AdTRAIL therapy. At least ten mice were included per group.

Evaluation of development of arthritis and joint damage. A caliper was used to determine the diameter of each paw of each mouse every day. Paw swelling was determined as the increase in diameter compared with the diameter at the initiation of the experiment. The severity of arthritis was graded according to the following scale: 0 , normal with no swelling and erythema and no increase in joint diameter; 1 , slight swelling and erythema with 0.1 - to 0.3 $\mathrm{mm}$ increase in joint diameter; 2 , swelling and erythema with 0.3 - to 0.6 - $\mathrm{mm}$ increase in joint diameter; 3 , extensive swelling and erythema with 0.6 - to $0.9-\mathrm{mm}$ increase in joint diameter; 4 , pronounced swelling and erythema with 0.9 - to $1.2-\mathrm{mm}$ increase in joint thickness or obvious joint destruction associated with visible joint deformity or ankylosis. Each limb was graded, resulting in a maximum clinical score of 16 per animal and expressed as the mean score on a given day. After sacrifice, the joints (knee, elbow, ankle, and wrist) were harvested, fixed in $10 \%$ formaldehyde/PBS for at least 24 hours, decalcified using EDTA for 4 weeks, sectioned at $5-\mu \mathrm{m}$ thickness, deparaffinized, and stained with $\mathrm{H} \& \mathrm{E}$ (Sigma-Aldrich, St. Louis, Missouri, USA).

Immunobistochemical staining of $T$ cell infiltration. The infiltration of $\mathrm{T}$ cells in the joint was determined by staining tissue sections with an anti-CD3 Ab. Quenching of endogenous peroxidase was performed by incubating tissue sections with $3 \% \mathrm{H}_{2} \mathrm{O}_{2}$ at RT for 15 minutes in a humidified chamber. After washing with PBS, tissue sections were incubated with $0.25 \%$ pepsin at $37^{\circ} \mathrm{C}$ for 30 minutes to reveal fixed Ag epitopes. Tissue sections were treated with blocking solution at RT for 30 minutes, followed by incubation with an HRP-conjugated anti-CD3 (DAKO Corp., Carpinteria, California, USA) at RT for 1 hour. Slides were incubated with a DAB staining kit (SK4100; Vector Laboratories, Burlingame, California, USA) for color visualization. Slides were counterstained by incubation with methyl green at $65^{\circ} \mathrm{C}$ for 3 minutes. Five fields were randomly selected for each joint, and the average number of infiltrating $T$ cells was determined by adding the total number of $T$ cells, then dividing by five to obtain the number of infiltrating $T$ cells per field of each joint. All four joints were evaluated, and the average number of infiltrating $\mathrm{T}$ cells per field per joint for each mouse was determined by adding the total number of infiltrating $T$ cells in all four joints, then dividing by four. At least five areas from each specimen were chosen randomly for assessment of the percentage of CD3-positive $\mathrm{T}$ cells in each specimen.

Analysis of the Ag-specific $T$ cell response after CII-DCAdTRAIL $+D O X$ treatment. Spleen $T$ cells from the five groups of mice were cocultured with syngeneic ( $\gamma$-irra- 
diated) DCs that were incubated with bovine CII (100 $\mu \mathrm{g} / \mathrm{ml}$ ) for 3 days as described; then the supernatants were collected and IFN- $\gamma$ in the supernatant was assayed by ELISA (Biosource International). After the assay, $1 \mu \mathrm{Ci}$ of ${ }^{3} \mathrm{H}$-thymidine, was added to the culture medium, the cells were harvested 16 hours later, and the incorporation of ${ }^{3} \mathrm{H}$-thymidine was determined using a scintillation counter.

ELISA quantification of autoantibody production. The concentration of the anti-CII Ab in the circulating blood was quantified using an ELISA assay in the five groups of mice. In brief, the serum levels of anti-mouse CII IgG were assayed using a mouse IgG anti-CII Ab assay ELISA kit (Chondrex Inc.) before treatment and at 12 weeks after primary immunization with bovine CII. A standard curve was produced using an anti-CII $\mathrm{Ab}$ provided with the ELISA kit.

Statistical analysis. The results are expressed as the mean plus or minus SEM. The two-tailed Student $t$ test was used for statistical analysis. ANOVA was used when more than two groups of samples were compared. A $P$ value of less than 0.05 was considered significant. The mean value of arthritis incidence in each treatment group is compared with that in the CIA-no treatment group by using the Mann-Whitney $U$ test.

\section{Results}

AdTRAIL-transfected DCs express murine TRAIL in a DOXinducible manner. Previously, we described depletion of $T$ cells with cell gene (APC-AdFasL) therapy. FasL was toxic to the APCs, however, resulting in autocrine apoptosis $(16,44)$. To develop a generally applicable strategy in which autocrine apoptosis of APCs is prevented, an AdTRAIL binary system was constructed (Figure 1a). One of the Ad's contain the TRAIL gene under the regulation of the TRE (45-47). The other Ad contains the rtTA under the regulation of the CMV promoter leading to high expression of rtTA $(43,48)$. This binary Ad system will be referred to as AdTRAIL. DCAdTRAIL exhibits DOX-inducible expression of TRAIL under the control of the DOX-inducible TRE.

To confirm the inducibility of biologically active TRAIL in this system, $5 \times 10^{6}$ immature DCs from the bone marrow of DBA/ $1 \mathrm{j}$ mice or TRAIL-sensitive HT1080 fibrosarcoma cells were transfected with either $50 \mathrm{pfu} / \mathrm{cell}$ of AdTRAIL or AdGFP. The cells were then stimulated with LPS to induce maturation, after which the cells were incubated with different concentrations of DOX for 24 hours. The transfection efficiency of the AdGFP-transfected DCs was evaluated using a fluorescence microscope. Nearly $90 \%$ of the AdGFP-transfected DCs were positive for GFP (Figure 1b). The expression of functional TRAIL capable of inducing apoptosis of transfected cells was then evaluated by an ATPlite assay. TRAIL expression on HT 1080 fibrosarcoma cells induced DOX-dependent killing of the cells (Figure 1c). There was no autocrine apoptosis of DCs after transfection with AdTRAIL, however, even at a high dose of DOX (Figure 1c). These results suggest that functional TRAIL could be expressed on the transfected cells and was not toxic to DCs.

Significantly decreased CII-induced arthritis after treatment with CII-DC-AdTRAIL+DOX in vivo. To demonstrate whether the treatment of CII-DC-AdTRAIL+DOX could prevent CII-induced arthritis, DCs from DBA/ $1 \mathrm{j}$ mice were transfected with AdTRAIL and then pulsed with bovine CII. The DCs were induced to maturation by stimulation with LPS. These DCs were then used to treat DBA/ $1 \mathrm{j}$ mice, commencing 2 weeks after the mice had been immunized with CII in CFA. As shown in Figure $2 \mathrm{a}$, mice received a total of four doses of these DCs $\left(5 \times 10^{6}\right.$ cells/dose $)$ over a 2 -week time period. At the same time, the mice (10 mice/group) received $2 \mathrm{mg} / \mathrm{ml}$ of DOX administered in drinking water with $4 \%$ sucrose or, as a control, $0.3 \%$ ethanol in water with $4 \%$ sucrose. The development of arthritis was assessed weekly up to 19 weeks of age (Figure 2a).

The administration of DOX alone in the range of $1.0-8.0 \mathrm{mg} / \mathrm{ml}$ confirmed that DOX alone had no effect on the development of arthritis (data not shown). The incidence of arthritis was significantly decreased in the group of mice treated with CII-DC-AdTRAIL+DOX $(P=0.003)$. Furthermore, the time of arthritis develop-
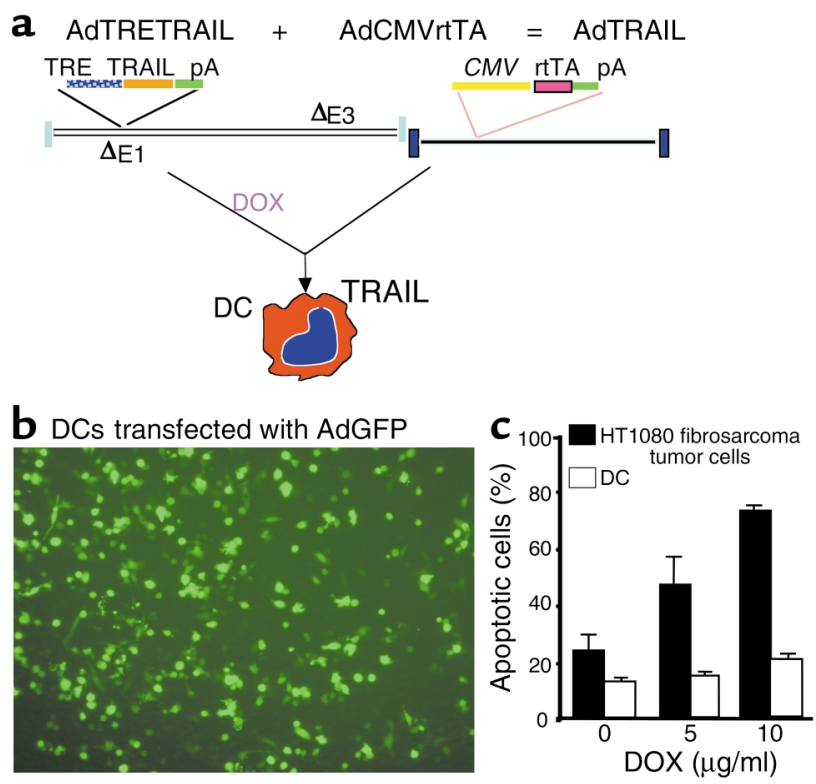

\section{Figure 1}

Inducible expression of murine TRAIL on DCs without induction of autocrine suicide. (a) A recombinant AdTRAIL was constructed as described in Methods. DCs from the bone marrow of DBA/ $1 \mathrm{j}$ mice were transfected with $50 \mathrm{pfu} / \mathrm{cell}$ of AdTRAIL and then incubated with different concentrations of DOX for 24 hours. The expression of functional TRAIL on the surface of the transfected cells was then evaluated by an ATPlite assay. (b) DCs from the bone marrow of DBA/1j mice were transfected with $50 \mathrm{pfu} / \mathrm{cell}$ of AdGFP, and the percentage of GFP+ DCs was quantitated 24 hours later under a fluorescence microscope. (c) TRAIL-sensitive HT1080 fibrosarcoma cells were transfected with $50 \mathrm{pfu} / \mathrm{cell}$ of AdTRAIL and then incubated with various concentrations of DOX for 24 hours. The expression of AdTRAIL in the target cells was tested using the ATPlite assay. Results are representative of three experiments. 


\section{Figure 2}

CII-DC-AdTRAIL+DOX treatment effectively prevents CII arthritis. (a) Diagram of treatment protocol as described in Methods. (b) CIA incidence in mice. Arthritis incidence in the different treatment groups was recorded every week until the mice were sacrificed. Shown are the statistically significant differences of the instances of mice that develop arthritis, comparing the CIA-no treatment group with each of the four treatment groups, as determined by the MannWhitney $U$ test. (c) DBA1j mice were challenged with $\mathrm{CI}$ and treated with CII-DC-AdTRAIL+DOX, CII-DC-AdTRAIL, CII-DC-AdGFP+DOX, or DCAdTRAIL+DOX. The mice were sacrificed at 19 weeks of age. Tissue sections were stained with H\&E. C, cartilage; $\mathrm{BM}$, bone marrow cavity; SLC, synovial lining cells; $\mathrm{SH}$, synovial hyperplasia. Original magnification, $\times 20$. (d) The joint severity score was quantitated using a double-blinded method. The severity score was divided into four levels using the scoring system described in Methods. Each bar represents the mean severity score from ten mice in each treatment group. The asterisk above the bar indicates treatment groups of mice that were significantly different from the $\mathrm{ClA}$-no treatment group of mice. ${ }^{*} P<0.05 ;{ }^{*} P<0.01$. a
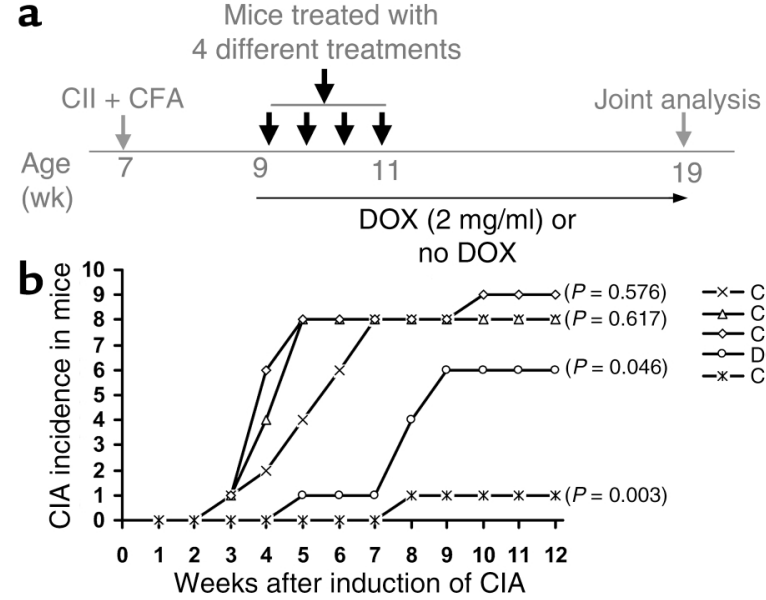

C

No treatment

CIA-no treatment
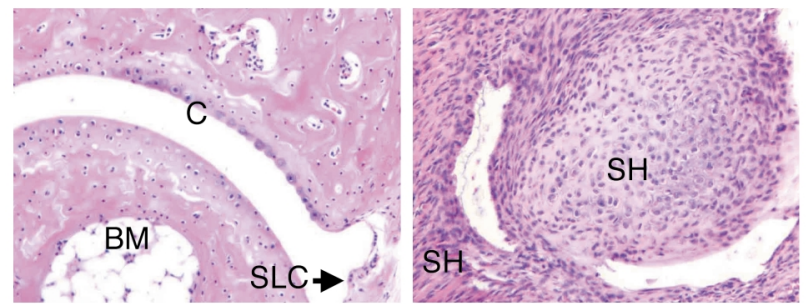

CII-DC-AdGFP+DOX

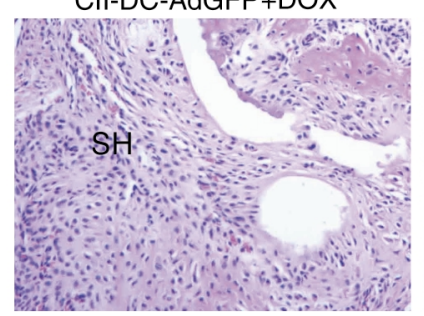

DC-AdTRAIL+DOX

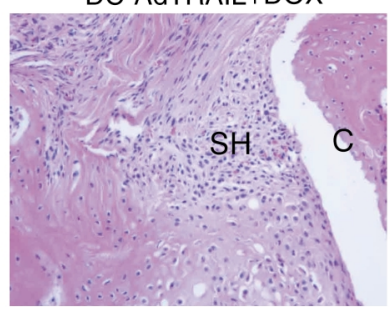

CII-DC-AdTRAIL+DOX
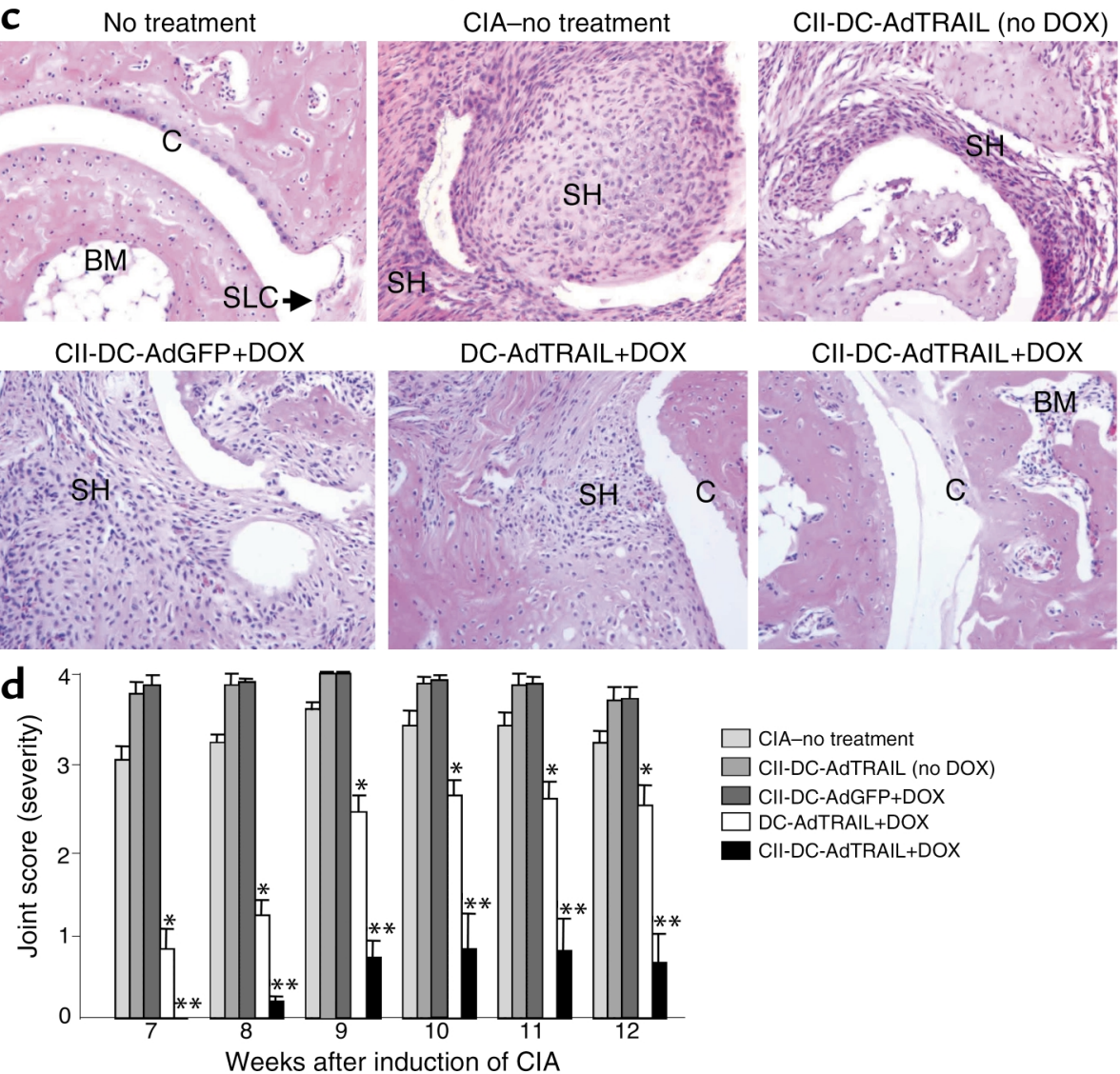

ment was significantly delayed in this group compared with the control groups (Figure 2b). Arthritis was also decreased and delayed in mice treated with DCAdTRAIL+DOX $(P=0.046)$, indicating that DCAdTRAIL+DOX in the absence of CII pulse can also diminish the incidence of CII arthritis. As anticipated, the control groups of mice treated with CII-DCAdTRAIL (no DOX) or CII-DC-AdGFP+DOX developed severe arthritis that was not significantly different from that in the CIA-no treatment group of mice (Figure 2b). Histological examination of the joints of the mice in control groups sacrificed at 19 weeks of age confirmed that histologic changes of severe arthritis were exhibited, with nearly all the joints showing pronounced syn- ovial hyperplasia, cartilage erosion, and ankylosis (Figure 2c). Moreover, the most severe morphologic change was observed in the control group of mice either untreated or treated with either CII-DC-AdTRAIL or (no DOX) CII-DC-AdGFP+DOX. Arthritis was partially decreased in mice treated with DC-AdTRAIL+DOX (no CII). The histologic features were the most significantly reduced in the group of mice treated with CIIDC-AdTRAIL+DOX. Compared with the CIA-no treatment group, there was a significant decrease in the joint severity score of mice treated with DC-AdTRAIL+DOX $(P<0.05)$ or with CII-DC-AdTRAIL+DOX $(P<0.01)$ (Figure 2d). Interestingly, control groups treated with CII-DC-AdTRAIL without DOX or CII-DC-AdGFP with 


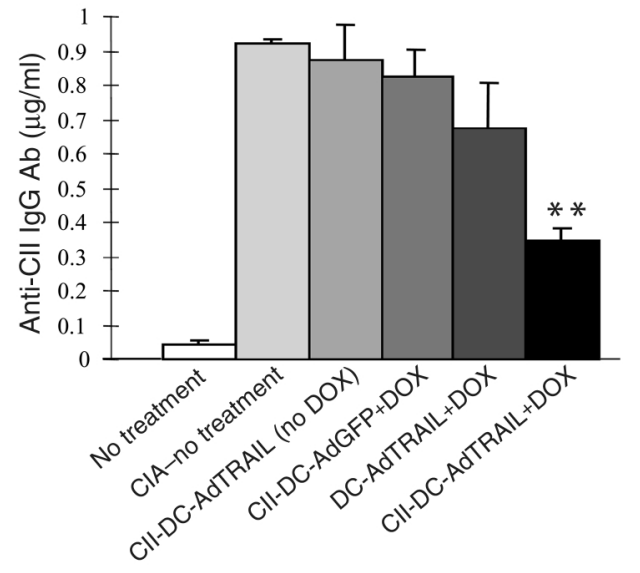

DOX resulted in a higher joint severity score compared with the CIA-no treatment group (Figure 2d). These results further confirm that optimal inhibition of CIA occurs in the presence of DCs pulsed with CII and requires expression of TRAIL induced by DOX.

Significantly decreased CII Ab production after treatment with CII-DC-AdTRAIL+DOX in vivo. To determine if CIIDC-AdTRAIL+DOX treatment is associated with a reduction of $\mathrm{CII} A \mathrm{~b}$ production, sera were collected at the time of sacrifice (19 weeks of age) and analyzed for the anti-CII Ab by an ELISA assay. The anti-CII IgG Ab levels in the sera of mice treated with CII-DCAdTRAIL+DOX were significantly lower than those in the control CIA-no treatment group of mice (Figure 3). This result suggests that CII-DC-AdTRAIL+DOX therapy can partially block anti-CII IgG Ab in the mice.

CII-DC-AdTRAIL $+D O X$ treatment inbibits $T$ cell infiltra-

\section{Figure 3}

CII-DC-AdTRAIL+DOX treatment partly decreased production of anti-CII Ab. The level of anti-CII Ab in the sera of mice receiving different treatments was measured by an ELISA assay. CII arthritis was induced in $\mathrm{DBA} / 1 \mathrm{j}$ mice, and different groups of mice were treated with CII-DC-AdTRAIL+DOX, CII-DC-AdTRAIL (no DOX), CII-DCAdGFP+DOX, or DC-AdTRAIL+DOX. The sera were collected at the time of sacrifice (19 weeks of age), and an ELISA assay was performed. CII-DC-AdTRAIL+DOX treatment significantly inhibited the sera level of $\mathrm{CII} A b$ in mice. Each bar represents the mean of the level of $\mathrm{Cll} \mathrm{Ab}$ from ten mice in each treatment group. ${ }^{*} P<0.01$.

tion in the joint. To determine whether systemic therapy with CII-DC-AdTRAIL+DOX could delete T cells and prevent the development of arthritis, in situ staining of the lesion areas using anti-CD3 $\mathrm{Ab}$ was performed. There was a significant decrease $(P<0.05)$ in the number of CD3-positive $\mathrm{T}$ cells infiltrating the synovium of mice treated with DC-AdTRAIL+DOX compared with mice treated with either CII-DC-AdTRAIL or CII-DCAdGFP+DOX, and almost no $T$ cells were observed in the joints of CII-DC-AdTRAIL+DOX-treated mice $(P<0.01)$ (Figure 4 , $a$ and $b)$. These results suggest that CII-DC-AdTRAIL+DOX is very effective in suppressing $\mathrm{T}$ cell infiltration in the joint.

Pretreatment of DCs with CII is required for elimination of CII-reactive T cells using DC-AdTRAIL therapy. We have demonstrated previously that pretreatment of APCs with CII pulse is required for elimination of the CII a No treatment

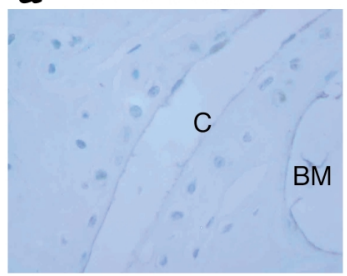

CII-DC-AdGFP+DOX

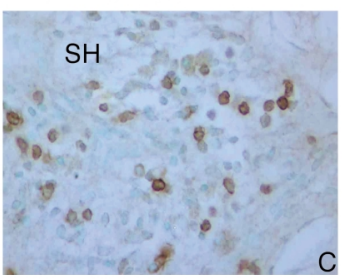

CIA-no treatment

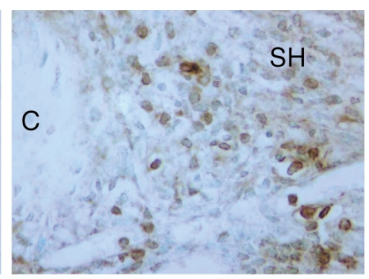

DC-AdTRAIL+DOX

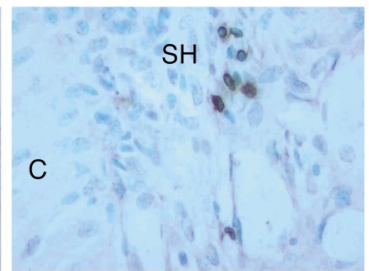

CII-DC-AdTRAIL (no DOX)

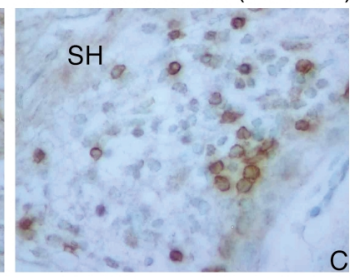

CII-DC-AdTRAIL+DOX

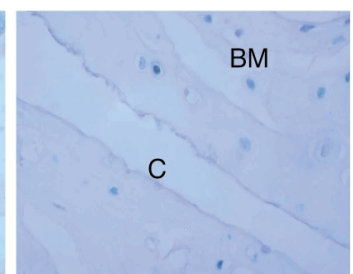

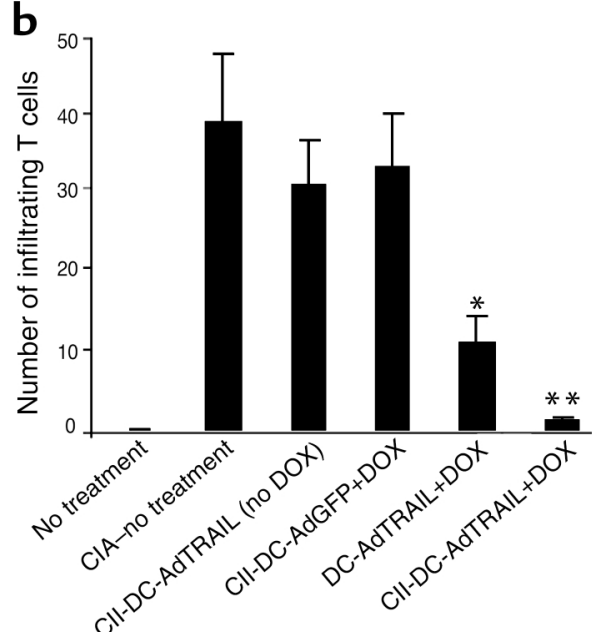

Figure 4

CII-DC-AdTRAIL+DOX treatment inhibits $T$ cell infiltration. (a) In situ staining of CD3 ${ }^{+} T$ cells in the joint area. DBA/ $1 \mathrm{j} \mathrm{mice} \mathrm{were} \mathrm{immunized}$ with CII and treated with CII-DC-AdTRAIL+DOX, CII-DC-AdTRAIL, CII-DC-AdGFP+DOX, or DC-AdTRAIL+DOX. The mice were sacrificed at 19 weeks of age, and the joints were sectioned and stained with anti-CD3 Ab. Dark brown cells indicate CD3-positive T cells. C, cartilage; BM, bone marrow cavity; $\mathrm{SH}$, synovial hyperplasia. Original magnification, $\times 40$. (b) Quantitative analysis of T cell infiltration. The numbers of CD3 positive $T$ cell infiltrating in the joint area were determined blindly as described in Methods. At least five areas from each specimen were chosen randomly to determine the numbers of T cell infiltrating in each specimen. T cell infiltration of the synovial area of CII-DC-AdTRAIL+DOX-treated mice was significantly reduced, compared with the CIA-no treatment group or other treatment groups. ${ }^{*} P<0.05 ;{ }^{*} P<0.01$. 

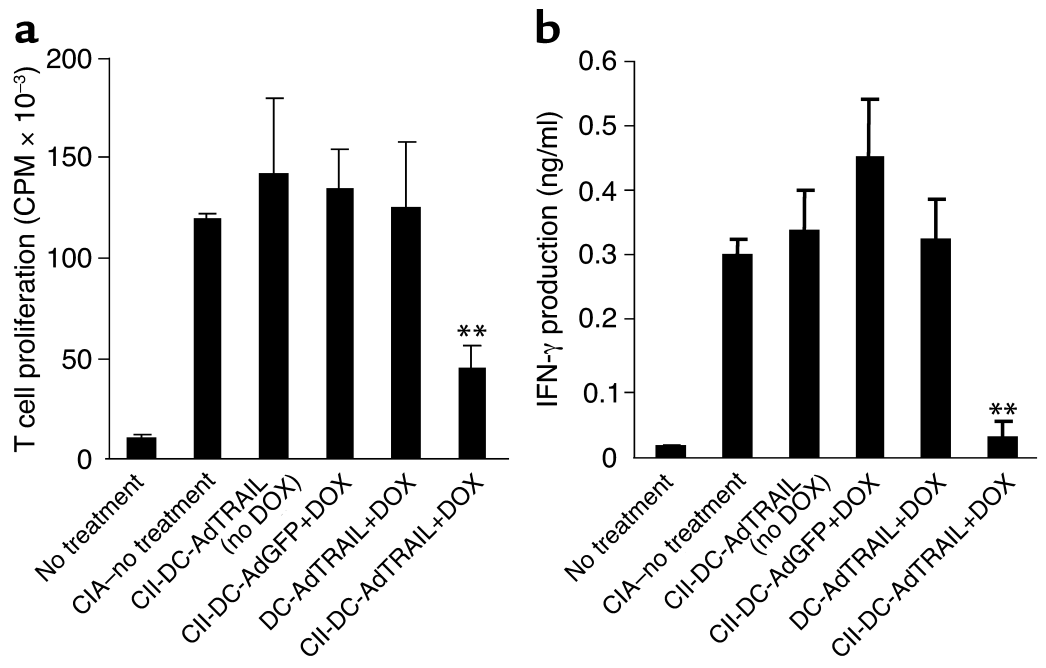

\section{Figure 5}

Cll-pulsed DCs are required for elimination of CII-reactive T cells. (a) Spleen T cells were isolated from the mice of various treatment groups shown at the bottom of the figure at the time of sacrifice ( 19 weeks of age) and were stimulated with $\gamma$-irradiated syngeneic spleen cells from DBA/1j mice that were pulsed with CII for 72 hours. The Cll-specific T cell-proliferative response was measured using a ${ }^{3} \mathrm{H}$-thymic uptake assay. The proliferation of the Cll-specific T cells was determined after an 18 -hour pulse of ${ }^{3} \mathrm{H}$-thymidine. The counts were determined using a scintillation counter. There was a statistically significant decrease of T cell proliferation in the CII-DC-AdTRAIL+DOX-treatment group, compared with the other treatment groups. ${ }^{*} P<0.01$. (b) Decreased secretion of IFN- $\gamma$ in the CII-DC-AdTRAIL+DOX-treatment group. IFN- $\gamma$ was determined in the supernatant at 72 hours after culture by an ELISA assay. The results represent the mean plus or minus SEM of duplicate cultures of ten mice per group analyzed separately. There was a statistically significant decrease of IFN- $\gamma$ production in the CII-DCAdTRAIL+DOX treatment group compared with the CIA-no treatment group and other treatment groups. ${ }^{*} P<0.01$.

response to T cells using APC-AdFasLp35Tet treatment to prevent arthritis (16). To determine if CIIDC-AdTRAIL could specifically eliminate CII-reactive $\mathrm{T}$ cells, single-cell suspensions were prepared from the spleens of mice in the different CIA treatment groups. The requirement of CII-pulsed DC-AdTRAIL treatment was demonstrated by an in vitro $T$ cell-proliferation assay and an IFN- $\gamma$-production assay. T cell proliferation was determined at 72 hours after stimulation by pulsing with ${ }^{3} \mathrm{H}$-thymidine 18 hours before harvest of the supernatants. There was a significant decrease $(P<0.01)$ in the $\mathrm{T}$ cell proliferative response as indicated by decreased ${ }^{3} \mathrm{H}$-thymidine uptake (Figure $5 \mathrm{a})$ and a significant decrease $(P<0.01)$ in the level of IFN- $\gamma$ production (Figure $5 \mathrm{~b}$ ) in the group of mice treated with CII-DC-AdTRAIL+DOX compared with other treatment groups. These results indicated that CII-loaded DC-AdTRAIL+DOX treatment is necessary to achieve high-specificity deletion of CIIreactive $\mathrm{T}$ cells and to inhibit development of CIIinduced arthritis.

DCs pulsed with AdTRAIL-induced apoptosis of T cells in the spleen. To determine if the CII-activated $\mathrm{T}$ cells are deleted in vivo, $\mathrm{DBA} / 1 \mathrm{j}$ mice were intraperitoneally injected with DCs transfected with either AdTRAIL or AdGFP followed by the addition of DOX in the drinking water. The trafficking of injected DCs was monitored by sectioning of the spleen, lymph nodes and the liver; apoptosis induction was quantified by in situ TUNEL staining at 48 hours after injection.Very strong GFP fluorescence was found in the spleens of mice treated with DC-AdGFP (Figure 6a). There was no GFP fluorescence, however, in the livers and lymph nodes of the same mice (data not shown) or in the spleens of mice injected with control DCs (Figure 6b). This indicates that the spleen is a primary site of migration of the injected DCs. In situ TUNEL staining of the spleen further showed that apoptotic $\mathrm{T}$ cells were detected in the spleens from the mice treated with DC-AdTRAIL+DOX (Figure $6 \mathrm{c}$ ). In contrast, there were no apoptotic $\mathrm{T}$ cells in the spleens from the mice treated with DCAdGFP+DOX (Figure 6d). These results indicate that functional TRAIL is expressed on the transfected DCs and induces apoptosis of $\mathrm{T}$ cells in the spleen.

\section{Discussion}

CII arthritis is a well-established mouse model for the study of erosive arthritis. This model has been used by many investigators to analyze the effects of either anti-T cell treatment $(4,9,14,23,49-52)$ or antiinflammatory treatment. This model is also used for defining the timing of therapeutic treatment. The present model shows that therapy initiated 2 weeks after primary immunization with CII can be used to ameliorate arthritis.

CII arthritis is dependent upon T cells. Myers and colleagues have cloned CII-specific $\mathrm{T}$ cells and have used these to transfer CII arthritis (2). This result showed that the processing of CII in the DBA/ $1 \mathrm{j}$ mouse leads to CII-specific T cells that can cause and transfer arthritis. Similarly, David and colleagues have shown that in an MHC human transgenic mouse model, peptides 
that react to human MHC DQ6 and DQ8 can induce arthritis with expansion of CII-specific T cells (53). In the present model, we have used this principle to limit the interaction between MHC-processed peptides and $T$ cells to specifically inhibit the development of arthritis. This was achieved by transfecting DCs with an Ad that expresses an inducible TRAIL. This results in specific induction and elimination of the T cells in the spleen (Figure 6) of the mouse, which prevents their migration into the joint. This is consistent with our previous results using a macrophage-derived APC-FasL cell gene therapy to prevent the development of arthritis in other murine arthritis models $(16,31)$. The present strategy is superior to our previous version, however, since DCs are more resistant to apoptosis than macrophages (54). In addition, TRAIL is a less potent cytolytic agent on normal cells than is FasL and has been used by other investigators to induce apoptosis in autoimmune disease, including arthritis (37, 38, 55-59). Therefore, the DC-AdTRAIL cell gene therapy does not need the coexpression of an inhibitor of apoptosis mechanism to prevent the autocrine apoptosis of the transfected APCs.

It has recently been shown that TRAIL can induce immune suppression by a mechanism other than deletion of autoreactive $T$ cells (38). In the experiments presented here, we have used a different system to express TRAIL. Therefore, in our experiments the conformation of TRAIL and binding to the TRAIL receptor may exhibit higher-affinity binding and signaling that results in in vivo $\mathrm{T}$ cell apoptosis. Also, in the present experiment, DC expression of TRAIL may play a role in the stabilization of the interaction of TRAIL with its receptor, leading to stronger signaling.

One key component of this DC-AdTRAIL cell gene therapy is the ability to regulate TRAIL expression. The most common way to regulate such molecules is by using an inducible promoter such as DOX. In the present experiments we have used a DOX-inducible promoter to enable expression of TRAIL only in the presence of DOX. In the absence of DOX, TRAIL expression is minimal, and the DCs do not induce detectable apoptosis. As the dose of DOX is increased, however, there was a gradual increase in expression of TRAIL and TRAIL-mediated apoptosis. A second advantage of using a DOX-inducible system is that mature, but not immature, DCs can be used to induce TRAIL expression. We reported previously that only mature DCs are resistant to apoptosis (44). Here, bone marrow-derived immature DCs were first transfected with AdTRAIL without DOX, after which DC maturation was induced by LPS before the addition of DOX. TRAIL expression was induced only in mature DCs that are resistant to TRAIL apoptosis. Therefore, the CII-DC-AdTRAIL+DOX cell gene therapy system described here meets the objectives of therapeutic treatment; that is, the therapeutic levels can be adjusted to achieve effective levels at optimal times without reaching toxic levels.
Another important component of the present cell gene therapy is the ability to increase killing specificity by pulsing DCs with CII to enhance apoptosis of CII-specific $\mathrm{T}$ cells. There is a statistically significant decrease in the severity of arthritis in mice treated with DC-AdTRAIL+DOX without pulsing with CII. The optimal decrease in arthritis, however, takes place in mice treated with CII-DC-AdTRAIL+DOX. We propose that the DC-AdTRAIL+DOX can have an ameliorating effect by nonspecific apoptosis of activated $T$ cells that play a role in the arthritis process.

It is of interest that the onset and severity of CII arthritis is made more severe by treating the mouse with DCs that have been pulsed with CII in the absence of the induction of TRAIL. This occurred for two treatment groups, the CII-DC-AdTRAIL (no DOX) and the CII-DC-AdGFP plus DOX. In both cases, the DCs expressed high levels of CII but did not induce $\mathrm{T}$ cell apoptosis since TRAIL is not expressed. Moreover, these results are consistent with results by Leung et al. (60), who showed that the presentation of collagenderived peptides by mature DCs was sufficient for induction of arthritis in DBA $/ 1 \mathrm{j}$ mice. Their experiments showed that DCs could be localized in lymph nodes 2 days after transfer, could induce a specific CD 4 $\mathrm{T}$ cell response, and that $\mathrm{T}$ cells expressed peripheral

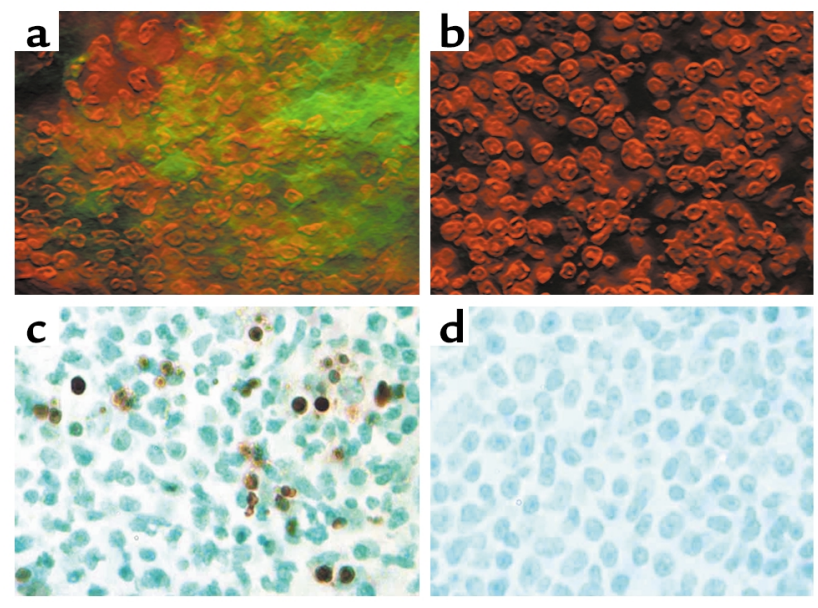

\section{Figure 6}

DCs migrate to the spleen. DCs were isolated from the bone marrow of DBA $/ 1 \mathrm{j}$ mice and cultured in the presence of GM-CSF for 4 days. They were then transfected with AdGFP at $50 \mathrm{pfu} / \mathrm{cell}$ for 18 hours in vitro before being intraperitoneally injected into CIIprimed DBA/1j mice. Forty-eight hours later, the spleen was collected and embedded with OCT. Ten-micrometer frozen sections were counterstained with Hoechst and examined by fluorescence microscope, then photographed. Original magnification, $\times 40$. (a) The spleen section from DBA $/ 1 \mathrm{j}$ mice injected with DC-AdGFP. (b) The spleen section from $D B A / 1 j$ mice injected with PBS. DCAdTRAIL+DOX induces activated T cell apoptosis in the spleen. The $\mathrm{DBA} / \mathrm{j}$ mice were treated with either DC-AdTRAIL+DOX or DCAdGFP+DOX, as described above. The spleen was then collected and embedded with paraffin. In situ TUNEL staining was performed. Original magnification, $\times 40$. (c) The spleen section from the mice treated with DC-AdTRAIL+DOX. (d) The spleen section from the mice treated with DC-AdGFP+DOX. 
cell nuclear Ag (PCNA). In those experiments, the DCs were injected into the footpad, similar to induction of CII arthritis, whereas in our experiments, intraperitoneal administration of the CII-pulsed DCs led predominantly to migration to the spleen. Future experiments will be performed to determine the optimal therapeutic levels for treatment using CII-DCAdTRAIL+DOX cell gene therapy.

The role of anti-CII Ab's in CII arthritis has been controversial. In the absence of CII Ab's, arthritis may progress, but generally has been found to be less severe $(50,61,62)$. This is consistent with our previous results using APC-FasL gene therapy where lower levels of CII Ab's are observed (16). The present experiments show that not only are autoreactive $\mathrm{T}$ cells eliminated by CIIDC-AdTRAIL+DOX cell gene therapy, but anti-CII Ab's are also diminished. It is noteworthy that the DC-Ad TRAIL+DOX group exhibited only a modest decrease of anti-CII Ab production. This is consistent with the decreased efficiency of treatment of arthritis for this group, compared with the CII-DC-AdTRAIL+DOX group. We propose that the DC-AdTRAIL+DOX treatment may induce apoptosis of T cells, since the DCs could directly interact with these T cells. B cells induced by initial immunization with CII, however, may continue to be present since they were induced to differentiate under the influence of $\mathrm{T}$ cell cytokines prior to treatment with DC-AdTRAIL+DOX. These results indicate that CII-DC-AdTRAIL+DOX cell gene therapy most likely eliminates helper $\mathrm{T}$ cells that promote the development of the anti-CII Ab response. Therefore, the present therapy eliminates both $\mathrm{T}$ cell components as well as the $\mathrm{B}$ cell and $\mathrm{Ab}$ components of $\mathrm{CII}$ arthritis.

In conclusion, CII-DC-AdTRAIL cell gene therapy is a highly beneficial method for specific suppression of an immune response. The cellular component, especially in the form of macrophages and DCs, can be used to process and present Ag's to specifically stimulate Agspecific T cells in the context of the appropriate MHC. This type of gene therapy uses the cytolytic capability of cytotoxic molecules such as FasL or TRAIL; once these genes are transfected into the APCs, they can be delivered to the appropriate site of interaction with $\mathrm{T}$ cells. The activation of the $\mathrm{T}$ cells by the CII-pulsed APCs results in in vivo CII-specific $T$ cell activation and specifically increases the susceptibility of these activated $\mathrm{T}$ cells to undergoing apoptosis. The presence of TRAIL in DCs under the regulation of DOX greatly eliminates the possibility that these cytotoxic cells can cause systemic damage. Therefore, we conclude that this inducible cell gene therapy is highly specific, yet nontoxic, and may be developed into an effective form for the treatment of arthritis or other $\mathrm{T}$ cell-mediated autoimmune diseases.

\section{Acknowledgments}

We thank Carol Humber for excellent secretarial work. This work was supported by grants from the Arthritis Foundation and a Birmingham VAMC Merit Review
Grant. H.-G. Zhang is supported by the Arthritis Foundation. J.D. Mountz is supported by a Birmingham VAMC Merit Review Grant.

1. Holmdahl, R., et al. 1989. Collagen induced arthritis as an experimental model for rheumatoid arthritis. Immunogenetics, pathogenesis and autoimmunity. APMIS. 97:575-584.

2. Myers, L.K., Rosloniec, E.F., Cremer, M.A., and Kang, A.H. 1997. Collagen-induced arthritis, an animal model of autoimmunity. Life Sci. 61:1861-1878.

3. Trucco, M., Robbins, P.D., Thomson, A.W., and Giannoukakis, N. 2002. Gene therapy strategies to prevent autoimmune disorders. Curr. Gene Ther. 2:341-354.

4. Robbins, P.D., Evans, C.H., and Chernajovsky, Y. 2003. Gene therapy for arthritis. Gene Ther. 10:902-911.

5. David, C.S. 1990. Genes for MHC, TCR and MIs determine susceptibility to collagen induced arthritis. APMIS. 98:575-584.

6. Holmdahl, R., et al. 1992. Homologous collagen-induced arthritis in rats and mice are associated with structurally different major histocompatibility complex DQ-like molecules. Eur. J. Immunol. 22:419-424.

7. Taneja, V., and David, C.S. 2000. Association of MHC and rheumatoid arthritis. Regulatory role of HLA class II molecules in animal models of RA: studies on transgenic/knockout mice. Arthritis Res. 2:205-207.

8. Holmdahl, R., Bockermann, R., Backlund, J., and Yamada, H. 2002. The molecular pathogenesis of collagen-induced arthritis in mice-a model for rheumatoid arthritis. Ageing Res. Rev. 1:135-147.

9. Backlund, J., et al. 2002. Glycosylation of type II collagen is of major importance for $\mathrm{T}$ cell tolerance and pathology in collagen-induced arthritis. Eur. J. Immunol. 32:3776-3784.

10. Banda, N.K., et al. 2002. Mechanisms of effects of complement inhibition in murine collagen-induced arthritis. Artbritis Rheum. 46:3065-3075.

11. Kafienah, W., Al-Fayez, F., Hollander, A.P., and Barker, M.D. 2003. Inhibition of cartilage degradation: a combined tissue engineering and gene therapy approach. Arthritis Rheum. 48:709-718.

12. Arai, K., et al. 1996. Extrathymic differentiation of resident $\mathrm{T}$ cells in the joints of mice with collagen-induced arthritis. J. Immunol. 157:5170-5177.

13. Corthay, A., Backlund, J., and Holmdahl, R. 2001. Role of glycopeptidespecific T cells in collagen-induced arthritis: an example how post-translational modification of proteins may be involved in autoimmune disease. Ann. Med. 33:456-465.

14. Nakajima, A., et al. 2001. Antigen-specific T cell-mediated gene therapy in collagen-induced arthritis. J. Clin. Invest. 107:1293-1301.

15. Tanaka, Y. 2001. The role of chemokines and adhesion molecules in the pathogenesis of rheumatoid arthritis. Drugs Today (Barc.). 37:477-484.

16. Zhang, H.G., et al. 2002. Depletion of collagen II-reactive T cells and blocking of B cell activation prevents collagen II-induced arthritis in DBA/1j mice. J. Immunol. 168:4164-4172.

17. Firestein, G.S. 2003. Evolving concepts of rheumatoid arthritis. Nature. 423:356-361.

18. Quayle, A.J., et al. 1993. Rheumatoid inflammatory T-cell clones express mostly Th1 but also Th2 and mixed (Th0-like) cytokine patterns. Scand. J. Immunol. 38:75-82.

19. Aarvak, T., Chabaud, M., Thoen, J., Miossec, P., and Natvig, J.B. 2000. Changes in the Th1 or Th2 cytokine dominance in the synovium of rheumatoid arthritis (RA): a kinetic study of the Th subsets in one unusual RA patient. Rheumatology (Oxford). 39:513-522.

20. Joosten, L.A., et al. 2003. Association of interleukin-18 expression with enhanced levels of both interleukin-1beta and tumor necrosis factor alpha in knee synovial tissue of patients with rheumatoid arthritis. Arthritis Rheum. 43:339-347.

21. Iwakura, Y. 2002. Roles of IL-1 in the development of rheumatoid arthritis: consideration from mouse models. Cytokine Growth Factor Rev. 13:341-355.

22. Eming, R., et al. 2002. Humanized mice as a model for rheumatoid arthritis. Arthritis Res. 3(Suppl.):S133-S140.

23. Tarner, I.H., et al. 2002. Retroviral gene therapy of collagen-induced arthritis by local delivery of IL-4. Clin. Immunol. 105:304-314.

24. Kadowaki, K.M., Matsuno, H., Tsuji, H., and Tunru, I. 1994. CD4+ T cells from collagen-induced arthritic mice are essential to transfer arthritis into severe combined immunodeficient mice. Clin. Exp. Immunol. 97:212-218.

25. Chu, C.Q., and Londei, M. 1996. Induction of Th2 cytokines and control of collagen-induced arthritis by nondepleting anti-CD4 Abs. J. Immunol. 157:2685-2689.

26. Mageed, R.A., Adams, G., Woodrow, D., Podhajcer, O.L., and Chernajovsky, Y. 1998. Prevention of collagen-induced arthritis by gene delivery of soluble p75 tumour necrosis factor receptor. Gene Ther. 5:1584-1592.

27. Mountz, J.D., Hsu, H.C., Matsuki, Y., and Zhang, H.G. 2001. Apoptosis and rheumatoid arthritis: past, present, and future directions. Curr. Rheumatol. Rep. 3:70-78. 
28. Van Laar, J.M., and Tyndall, A. 2003. Intense immunosuppression and stem-cell transplantation for patients with severe rheumatic autoimmune disease: a review. Cancer Control. 10:57-65.

29. Zhang, H.G., et al. 1999. Induction of specific T cell tolerance by Fas ligand-expressing antigen-presenting cells. J. Immunol. 162:1423-1430.

30. Zhang, H.G., et al. 2000. Gene therapy that inhibits nuclear translocation of nuclear factor kappaB results in tumor necrosis factor alpha-induced apoptosis of human synovial fibroblasts. Arthritis Rheum. 43:1094-1105.

31. Hsu, H.C., et al. 2001. Defective Fas ligand-mediated apoptosis predisposes to development of a chronic erosive arthritis subsequent to Mycoplasma pulmonis infection. Arthritis Rheum. 44:2146-2159.

32. Baetu, T.M., and Hiscott, J. 2002. On the TRAIL to apoptosis. Cytokine Growth Factor Rev. 13:199-207.

33. LeBlanc, H.N., and Ashkenazi, A. 2003. Apo2L/TRAIL and its death and decoy receptors. Cell Death Differ. 10:66-75.

34. MacFarlane, M. 2003. TRAIL-induced signalling and apoptosis. Toxicol. Lett. 139:89-97.

35. Strater, J., et al. 2002. Expression of TRAIL and TRAIL receptors in colon carcinoma: TRAIL-R1 is an independent prognostic parameter. Clin. Cancer Res. 8:3734-3740.

36. Zauli, G., et al. 2003. Tumor necrosis factor-related apoptosis-inducing ligand (TRAIL) sequentially upregulates nitric oxide and prostanoid production in primary human endothelial cells. Circ. Res. 92:732-740.

37. Lamhamedi-Cherradi, S.E., Zheng, S.J., Maguschak, K.A., Peschon, J., and Chen, Y.H. 2003. Defective thymocyte apoptosis and accelerated autoimmune diseases in TRAIL-/- mice. Nat. Immunol. 4:255-260.

38. Song, K., et al. 2000. Tumor necrosis factor-related apoptosis-inducing ligand (TRAIL) is an inhibitor of autoimmune inflammation and cell cycle progression. J. Exp. Med. 191:1095-1104.

39. Yao, Q., et al. 2003. Intra-articular adenoviral-mediated gene transfer of trail induces apoptosis of arthritic rabbit synovium. Gene Ther. 10:1055-1060.

40. Zhang, H.G., et al. 2000. Adeno-associated virus production of soluble tumor necrosis factor receptor neutralizes tumor necrosis factor alpha and reduces arthritis. Hum. Gene Ther. 11:2431-2442.

41. Mountz, J.D., and Zhang, H.G. 2001. Regulation of apoptosis of synovial fibroblasts. Curr. Dir. Autoimmun. 3:216-239.

42. He, T.C., et al. 1998. A simplified system for generating recombinant adenoviruses. Proc. Natl. Acad. Sci. U. S. A. 95:2509-2514.

43. Harding, T.C., Geddes, B.J., Murphy, D., Knight, D., and Uney, J.B. 1998. Switching transgene expression in the brain using an adenoviral tetracycline-regulatable system. Nat. Biotechnol. 16:553-555.

44. Hoves, S., et al. 2003. Mature but not immature Fas ligand (CD95L)transduced human monocyte-derived dendritic cells are protected from Fas-mediated apoptosis and can be used as killer APC. J. Immunol. 170:5406-5413

45. Gossen, M., and Bujard, H. 1992. Tight control of gene expression in mammalian cells by tetracycline-responsive promoters. Proc. Natl. Acad. Sci. U. S. A. 89:5547-5551.

46. Gossen, M., et al. 1995. Transcriptional activation by tetracyclines in mammalian cells. Science. 268:1766-1769.

47. Baron, U., and Bujard, H. 2000. Tet repressor-based system for regulated gene expression in eukaryotic cells: principles and advances. Methods Enzymol. 327:401-421.

48. Bohl, D., Salvetti, A., Moullier, P., and Heard, J.M. 1998. Control of erythropoietin delivery by doxycycline in mice after intramuscular injection of adeno-associated vector. Blood. 92:1512-1517.

49. Kim, S.H., Kim, S., Oligino, T.J., and Robbins, P.D. 2002. Effective treatment of established mouse collagen-induced arthritis by systemic administration of dendritic cells genetically modified to express FasL. Mol. Ther. 6:584-590.

50. Wang, D., Hill, J.A., Jevnikar, A.M., Cairns, E., and Bell, D.A. 2002. Induction of transient arthritis by the adoptive transfer of a collagen II specific Th1 clone to HLA-DR4 (B1*0401) transgenic mice. J. Autoimmun. 19:37-43.

51. Shin, S.S., et al. 2003. Suppressive effects of PG201, an ethanol extract from herbs, on collagen-induced arthritis in mice. Rheumatology (Oxford). 42:665-672.

52. Myers, L.K., et al. 2002. Peptide-induced suppression of collagen-induced arthritis in HLA-DR1 transgenic mice. Arthritis Rheum. 46:3369-3377.

53. Krco, C.J., et al. 1999. Identification of T cell determinants on human type II collagen recognized by HLA-DQ8 and HLA-DQ6 transgenic mice. J. Immunol. 163:1661-1665.

54. Ashany, D., Savir, A., Bhardwaj, N., and Elkon, K.B. 1999. Dendritic cells are resistant to apoptosis through the Fas (CD95/APO-1) pathway. J. Immunol. 163:5303-5311.

55. Zhou, T., Mountz, J.D., and Kimberly, R.P. 2002. Immunobiology of tumor necrosis factor receptor superfamily. Immunol Res. 26:323-336.

56. Zhang, H.G., et al. 2002. Hepatic DR5 induces apoptosis and limits adenovirus gene therapy product expression in the liver. J. Virol. 76:5692-5700.

57. Gardnerova, M., Blanque, R., and Gardner, C.R. 2000. The use of TNF family ligands and receptors and agents which modify their interaction as therapeutic agents. Curr. Drug Targets. 1:327-364.

58. Ichikawa, K., et al. 2001. Tumoricidal activity of a novel anti-human DR5 monoclonal antibody without hepatocyte cytotoxicity. Nat. Med. 7:954-960.

59. Matsumura, R., et al. 2002. Expression of TNF-related apoptosis inducing ligand (TRAIL) on infiltrating cells and of TRAIL receptors on salivary glands in patients with Sjogren's syndrome. Clin. Exp. Rheumatol. 20:791-798.

60. Leung, B.P., et al. 2002. A novel dendritic cell-induced model of erosive inflammatory arthritis: distinct roles for dendritic cells in T cell activation and induction of local inflammation. J. Immunol. 169:7071-7077.

61. Chu, C.Q., and Londei, M. 1999. Differential activities of immunogenic collagen type II peptides in the induction of nasal tolerance to collageninduced arthritis. J. Autoimmun. 12:35-42.

62. Niizawa, A., et al. 2003. Clinical and immunomodulatory effects of funboi, an herbal medicine, on collagen-induced arthritis in vivo. Clin. Exp. Rheumatol. 21:57-62. 